JOURNAL OF THE

CHUNGCHEONG MATHEMATICAL SOCIETY

Volume 26, No. 4, November 2013

http://dx.doi.org/10.14403/jcms.2013.26.4.887

\title{
STABILITY OF A GENERALIZED POLYNOMIAL FUNCTIONAL EQUATION OF DEGREE 2 IN NON-ARCHIMEDEAN NORMED SPACES
}

\author{
Chang-Ju LeE* AND YAnG-Hi LeE**
}

\begin{abstract}
In this paper, we investigate the stability for the functional equation

$$
f(3 x+y)-3 f(2 x+y)+3 f(x+y)-f(y)=0
$$
\end{abstract}

in the sense of M. S. Moslehian and Th. M. Rassias.

\section{Introduction}

The stability problem of the functional equation was formulated by S. M. Ulam [16] in 1940. D. H. Hyers [4], T. Aoki [1] and Th. M. Rassias [15] made important role to study the stability of the functional equation. During the last decades, the stability problems of functional equations have been extensively investigated by a number of mathematicians, see [2],[3], [6]-[13].

By a non-Archimedean field, we mean a field $\mathbb{K}$ equipped with a function (valuation) $|\cdot|$ from $\mathbb{K}$ into $[0, \infty)$ such that $|r|=0$ if and only if $r=0,|r s|=|r||s|$, and $|r+s| \leq \max \{|r|,|s|\}$ for all $r, s \in \mathbb{K}$. Clearly $|1|=|-1|$ and $|n| \leq 1$ for all $n \in \mathbb{N}$. Let $X$ be a vector space over a scalar field $\mathbb{K}$ with a non-Archimedean non- trivial valuation $|\cdot|$. A function $\|\cdot\|: X \rightarrow \mathbb{R}$ is a non-Archimedean norm (valuation) if it satisfies the following conditions:

(i) $\|x\|=0$ if and only if $x=0$;

(ii) $\|r x\|=|r|\|x\|(r \in \mathbb{K}, x \in X)$;

Received September 24, 2013; Accepted November 04, 2013.

2010 Mathematics Subject Classification: Primary 46S40, 39B52.

Key words and phrases: generalized polynomial functional equation of degree 2, non-Archimedean normed space, stability of functional equation.

Correspondence should be addressed to Yang-Hi Lee, lyhmzi@gjue.ac.kr.

This work was supported by Gongju National University of Education Grant. 
(iii) the strong triangle inequality, namely,

$$
\|x+y\| \leq \max \{\|x\|,\|y\|\}
$$

for all $x, y \in X$ and $r \in \mathbb{K}$.

Then $(X,\|\cdot\|)$ is called a non-Archimedean normed space. Due to the fact that

$$
\left\|x_{n}-x_{m}\right\| \leq \max \left\{\left\|x_{j+1}-x_{j}\right\|: m \leq j \leq n-1\right\}(n>m),
$$

a sequence $\left\{x_{n}\right\}$ is Cauchy if and only if $\left\{x_{n+1}-x_{n}\right\}$ converges to zero in a non-Archimedean normed space. By a complete non-Archimedean space, we mean one in which every Cauchy sequence is convergent.

Recently M. S. Moslehian and Th. M. Rassias [14] discussed the Hyers-Ulam stability of the Cauchy functional equation $f(x+y)=f(x)+$ $f(y)$ and the quadratic functional equation $f(x+y)+f(x-y)-2 f(x)-$ $2 f(y)=0$ in non-Archimedean normed spaces.

Now we consider the generalized polynomial functional equation of degree 2

$$
f(3 x+y)-3 f(2 x+y)+3 f(x+y)-f(y)=0
$$

whose solution is called a general quadratic mapping. In 2009, the second author [9] obtained a stability of the generalized polynomial functional equation of degree 2 by taking and composing an additive mapping $A$ and a quadratic mapping $Q$ to prove the existence of a general quadratic function $F$ which is close to the given function $f$. In his processing, $A$ is approximate to the odd part $\frac{f(x)-f(-x)}{2}$ of $f$ and $Q$ is close to the even part $\frac{f(x)+f(-x)}{2}-f(0)$ of it, respectively.

In this paper, we get a general stability result of the generalized polynomial functional equation of degree 2 in non-Archimedean normed spaces.

\section{Stability of the generalized polynomial functional equa- tion of degree 2}

In this section, we prove the generalized Hyers-Ulam stability of the generalized polynomial functional equation of degree 2 . Throughout this section, we assume that $X$ is a non-Archimedean normed space and $Y$ is a complete non-Archimedean space.

For a given mapping $f: X \rightarrow Y$, we use the abbreviation

$$
D f(x, y):=f(3 x+y)-3 f(2 x+y)+3 f(x+y)-f(y)
$$


for all $x, y \in X$.

Lemma 2.1. (Lemma 3.1 in [5]) If $f: X \rightarrow Y$ is a mapping such that $D f(x, y)=0$ for all $x, y \in X \backslash\{0\}$, then $f$ is a general quadratic mapping.

Theorem 2.2. Let $\varphi:(X \backslash\{0\})^{2} \rightarrow[0, \infty)$ be a function such that

$$
\lim _{n \rightarrow \infty} \frac{\varphi\left(2^{n} x, 2^{n} y\right)}{|4|^{n}}=0
$$

for all $x, y \in X \backslash\{0\}$ and let for each $x \in X \backslash\{0\}$ the limit

$$
\lim _{n \rightarrow \infty} \max _{0 \leq j<n}\left\{\frac{\varphi\left(2^{j} x,-2^{j} x\right)}{|2| \cdot|4|^{j+1}}, \frac{\varphi\left(-2^{j} x, 2^{j} x\right)}{|2| \cdot|4|^{j+1}}\right\},
$$

denoted by $\tilde{\varphi}(x)$, exists. Suppose that $f: X \rightarrow Y$ is a mapping satisfying the inequality

$$
\|D f(x, y)\| \leq \varphi(x, y)
$$

for all $x, y \in X \backslash\{0\}$. Then there exists a unique general quadratic mapping $T: X \rightarrow Y$ such that

$$
\|f(x)-T(x)\| \leq \tilde{\varphi}(x)
$$

for all $x \in X \backslash\{0\}$ with $T(0)=f(0)$. In particular, $T$ is given by

$$
\begin{aligned}
T(x)= & \lim _{n \rightarrow \infty} \frac{f\left(2^{n} x\right)+f\left(-2^{n} x\right)-2 f(0)}{2 \cdot 4^{n}} \\
& +\lim _{n \rightarrow \infty} \frac{f\left(2^{n} x\right)-f\left(-2^{n} x\right)}{2^{n+1}}+f(0)
\end{aligned}
$$

for all $x \in X$.

Proof. Let $J_{n} f: X \rightarrow Y$ be a mapping defined by

$$
J_{n} f(x)=\frac{f\left(2^{n} x\right)+f\left(-2^{n} x\right)-2 f(0)}{2 \cdot 4^{n}}+\frac{f\left(2^{n} x\right)-f\left(-2^{n} x\right)}{2^{n+1}}+f(0)
$$

for all $x \in X$ and all $n \in \mathbb{N}$. Notice that $J_{0} f(x)=f(x)$ and 


$$
\begin{aligned}
\left\|J_{j} f(x)-J_{j+1} f(x)\right\|= & \|-\frac{D f\left(2^{j} x,-2^{j} x\right)}{2 \cdot 4^{j+1}}-\frac{D f\left(-2^{j} x, 2^{j} x\right)}{2 \cdot 4^{j+1}} \\
& -\frac{D f\left(2^{j} x,-2^{j} x\right)}{2^{j+2}}+\frac{D f\left(-2^{j} x, 2^{j} x\right)}{2^{j+2}} \| \\
\leq & \max \left\{\frac{\left\|D f\left(2^{j} x,-2^{j} x\right)\right\|}{|2| \cdot|4|^{j+1}}, \frac{\left\|D f\left(-2^{j} x, 2^{j} x\right)\right\|}{|2| \cdot|4|^{j+1}},\right. \\
& \left.\frac{\left\|D f\left(2^{j} x,-2^{j} x\right)\right\|}{|2|^{j+2}}, \frac{\left\|D f\left(-2^{j} x, 2^{j} x\right)\right\|}{|2|^{j+2}}\right\} \\
(2.5) \leq & \max \left\{\frac{\varphi\left(2^{j} x,-2^{j} x\right)}{|2| \cdot|4|^{j+1}}, \frac{\varphi\left(-2^{j} x, 2^{j} x\right)}{|2| \cdot|4|^{j+1}}\right\}
\end{aligned}
$$

for all $x \in X \backslash\{0\}$ and all $j \geq 0$. It follows from (2.5) and (2.1) that the sequence $\left\{J_{n} f(x)\right\}$ is Cauchy for all $x \in X \backslash\{0\}$. Since $Y$ is complete and $J_{n} f(0)=f(0)$ for all $n \in \mathbb{N}$, we conclude that $\left\{J_{n} f(x)\right\}$ is convergent for all $x \in X$. Set

$$
T(x):=\lim _{n \rightarrow \infty} J_{n} f(x) .
$$

One can show that

$$
\begin{aligned}
\left\|J_{n} f(x)-f(x)\right\| & =\left\|\sum_{j=0}^{n-1} J_{j} f(x)-J_{j+1} f(x)\right\| \\
& \leq \max _{0 \leq j<n}\left\{\frac{\varphi\left(2^{j} x,-2^{j} x\right)}{|2| \cdot|4|^{j+1}}, \frac{\varphi\left(-2^{j} x, 2^{j} x\right)}{|2| \cdot|4|^{j+1}}\right\}
\end{aligned}
$$

for all $n \in N$ and all $x \in X \backslash\{0\}$. By taking $n$ to approach infinity in (2.6) and using (2.2) one obtains (2.4). Replacing $x$ and $y$ by $2^{n} x$ and $2^{n} y$, respectively, in $(2.3)$ we get

$$
\begin{aligned}
&\left\|D J_{n} f(x, y)\right\|= \| \frac{D f\left(2^{n} x, 2^{n} y\right)-D f\left(-2^{n} x,-2^{n} y\right)}{2^{n+1}} \\
&+\frac{D f\left(2^{n} x, 2^{n} y\right)+D f\left(-2^{n} x,-2^{n} y\right)}{2^{2 n+1}} \| \\
& \leq \max \left\{\frac{\varphi\left(2^{n} x, 2^{n} y\right)}{|2|^{n+1}}, \frac{\varphi\left(-2^{n} x,-2^{n} y\right)}{|2|^{n+1}},\right. \\
&\left.\frac{\varphi\left(2^{n} x, 2^{n} y\right)}{|2| \cdot|4|^{n}}, \frac{\varphi\left(-2^{n} x,-2^{n} y\right)}{|2| \cdot|4|^{n}}\right\}
\end{aligned}
$$

for all $x, y \in X \backslash\{0\}$ and all $n \in \mathbb{N}$. Taking the limit as $n \rightarrow \infty$ and using (2.1) and Lemma 2.1 we get $D T(x, y)=0$ for all $x, y \neq 0$ and so $T$ is a 
general quadratic mapping. Now we are going to prove the uniqueness of $T$. If $T^{\prime}$ is another general quadratic mapping satisfying (2.4) with $T^{\prime}(0)=f(0)$, then

$$
\begin{aligned}
T^{\prime}(x)= & \sum_{j=0}^{k-1}\left(-\frac{D T^{\prime}\left(2^{j} x,-2^{j} x\right)}{2 \cdot 4^{j+1}}-\frac{D T^{\prime}\left(-2^{j} x, 2^{j} x\right)}{2 \cdot 4^{j+1}}\right. \\
& \left.-\frac{D T^{\prime}\left(2^{j} x,-2^{j} x\right)}{2^{j+2}}+\frac{D T^{\prime}\left(-2^{j} x, 2^{j} x\right)}{2^{j+2}}\right)+J_{k} T^{\prime}(x) \\
= & J_{k} T^{\prime}(x)
\end{aligned}
$$

for any $k \in N$ and so

$$
\begin{aligned}
& \left\|T(x)-T^{\prime}(x)\right\| \\
& =\lim _{k \rightarrow \infty}\left\|J_{k} T(x)-J_{k} T^{\prime}(x)\right\| \\
& \leq \lim _{k \rightarrow \infty} \max \left\{\left\|J_{k} T(x)-J_{k} f(x)\right\|,\left\|J_{k} f(x)-J_{k} T^{\prime}(x)\right\|\right\} \\
& \leq \lim _{k \rightarrow \infty}|2|^{-2 k-1} \max \left\{\left\|T\left(2^{k} x\right)-f\left(2^{k} x\right)\right\|,\left\|T\left(-2^{k} x\right)-f\left(-2^{k} x\right)\right\|,\right. \\
& \left.\qquad\left\|f\left(2^{k} x\right)-T^{\prime}\left(2^{k} x\right)\right\|,\left\|f\left(-2^{k} x\right)-T^{\prime}\left(-2^{k} x\right)\right\|\right\} \\
& \leq \lim _{k \rightarrow \infty} \lim _{n \rightarrow \infty} \max _{k \leq j<n+k}\left\{\frac{\varphi\left(2^{j} x,-2^{j} x\right)}{|4|^{j+2}}, \frac{\varphi\left(-2^{j} x, 2^{j} x\right)}{|4|^{j+2}}\right\} \\
& =0
\end{aligned}
$$

for all $x \in X \backslash\{0\}$. Since $T(0)=f(0)=T^{\prime}(0)$, we get $T(x)=T^{\prime}(x)$ for all $x \in X$. This completes the proof of the uniqueness of $T$.

Corollary 2.3. Let $2<r$ be a real number and $|2|<1$. If $f: X \rightarrow$ $Y$ satisfies the inequality

$$
\|D f(x, y)\| \leq \theta\left(\|x\|^{r}+\|y\|^{r}\right)
$$

for all $x, y \in X$, then there exists a unique general quadratic mapping $T: X \rightarrow Y$ such that

$$
\|f(x)-T(x)\| \leq 2 \theta|2|^{-3}\|x\|^{r}
$$

for all $x \in X$ with $T(0)=f(0)$.

Proof. Let $\varphi(x, y)=\theta\left(\|x\|^{r}+\|y\|^{r}\right)$. Since $|2|<1$ and $r-2>0$,

$$
\lim _{n \rightarrow \infty}|4|^{-n} \varphi\left(2^{n} x, 2^{n} y\right)=\lim _{n \rightarrow \infty}|2|^{n(r-2)} \varphi(x, y)=0
$$

for all $x, y \in X$. Therefore the conditions of Theorem 2.2 are fulfilled and it is easy to see that $\tilde{\varphi}(x)=2 \theta|2|^{-3}\|x\|^{r}$. By Theorem 2.2 there is 
a unique general quadratic mapping $T: X \rightarrow Y$ satisfying (2.7) with $T(0)=f(0)$.

Theorem 2.4. Let $\varphi:(X \backslash\{0\})^{2} \rightarrow[0, \infty)$ be a function such that

$$
\lim _{n \rightarrow \infty}|2|^{n} \varphi\left(2^{-n} x, 2^{-n} y\right)=0
$$

for all $x, y \in X \backslash\{0\}$ and let for each $x \in X \backslash\{0\}$ the limit

$$
\lim _{n \rightarrow \infty} \max _{0 \leq j<n}\left\{|2|^{j-1} \varphi\left(\frac{x}{2^{j+1}}, \frac{-x}{2^{j+1}}\right),|2|^{j-1} \varphi\left(\frac{-x}{2^{j+1}}, \frac{x}{2^{j+1}}\right)\right\},
$$

denoted by $\tilde{\varphi}(x)$, exists. Suppose that $f: X \rightarrow Y$ is a mapping satisfying the inequality

$$
\|D f(x, y)\| \leq \varphi(x, y)
$$

for all $x, y \in X \backslash\{0\}$. Then there exists a unique general quadratic mapping $T: X \rightarrow Y$ such that

$$
\|f(x)-T(x)\| \leq \tilde{\varphi}(x)
$$

for all $x \in X \backslash\{0\}$ with $T(0)=f(0)$. In particular, $T$ is given by

$$
\begin{aligned}
T(x)= & \lim _{n \rightarrow \infty} \frac{4^{n}}{2}\left(f\left(\frac{x}{2^{n}}\right)+f\left(\frac{-x}{2^{n}}\right)-2 f(0)\right) \\
& +\lim _{n \rightarrow \infty} 2^{n-1}\left(f\left(\frac{x}{2^{n}}\right)-f\left(\frac{-x}{2^{n}}\right)\right)+f(0)
\end{aligned}
$$

for all $x \in X$.

Proof. Let $J_{n} f: X \rightarrow Y$ be a mapping defined by

$$
\begin{aligned}
J_{n} f(x)= & \frac{4^{n}}{2}\left(f\left(2^{-n} x\right)+f\left(-2^{-n} x\right)-2 f(0)\right) \\
& +2^{n-1}\left(f\left(\frac{x}{2^{n}}\right)-f\left(-\frac{x}{2^{n}}\right)\right)+f(0)
\end{aligned}
$$

for all $x \in X$ and $n \in \mathbb{N}$. Notice that $J_{0} f(x)=f(x)$ and

$$
\begin{aligned}
\| J_{j} f(x) & -J_{j+1} f(x) \| \\
= & \|\left(2^{2 j-1}+2^{j-1}\right) \operatorname{Df}\left(\frac{x}{2^{j+1}}, \frac{-x}{2^{j+1}}\right) \\
& +\left(2^{2 j-1}-2^{j-1}\right) \operatorname{Df}\left(\frac{-x}{2^{j+1}}, \frac{x}{2^{j+1}}\right) \| \\
\leq & \max \left\{|2|^{j-1} \varphi\left(\frac{x}{2^{j+1}}, \frac{-x}{2^{j+1}}\right),|2|^{j-1} \varphi\left(\frac{-x}{2^{j+1}}, \frac{x}{2^{j+1}}\right)\right\}
\end{aligned}
$$


for all $x \in X \backslash\{0\}$ and all $j \geq 0$. It follows from (2.12) and (2.8) that the sequence $\left\{J_{n} f(x)\right\}$ is Cauchy for all $x \in X \backslash\{0\}$. Since $Y$ is complete and $J_{n} f(0)=f(0)$, we conclude that $\left\{J_{n} f(x)\right\}$ is convergent for all $x \in X$. Set

$$
T(x):=\lim _{n \rightarrow \infty} J_{n} f(x)
$$

for all $x \in X$. Using induction one can show that

$$
\begin{aligned}
& \left\|J_{n} f(x)-f(x)\right\| \\
& \leq \max _{0 \leq j<n}\left\{|2|^{j-1} \varphi\left(\frac{x}{2^{j+1}}, \frac{-x}{2^{j+1}}\right),|2|^{j-1} \varphi\left(\frac{-x}{2^{j+1}}, \frac{x}{2^{j+1}}\right)\right\}
\end{aligned}
$$

for all $n \in N$ and all $x \in X \backslash\{0\}$. By taking $n$ to approach infinity in (2.13) and using (2.9) one obtains (2.11). Replacing $x$ and $y$ by $2^{-n} x$ and $2^{-n} y$, respectively, in (2.10) we get

$$
\begin{aligned}
\left\|D J_{n} f(x, y)\right\|= & \| 2^{n-1} D f\left(\frac{x}{2^{n}}, \frac{y}{2^{n}}\right)-2^{n-1} D f\left(\frac{-x}{2^{n}}, \frac{-y}{2^{n}}\right) \\
& +2^{2 n-1} D f\left(\frac{x}{2^{n}}, \frac{y}{2^{n}}\right)+2^{2 n-1} D f\left(\frac{-x}{2^{n}}, \frac{-y}{2^{n}}\right) \| \\
\leq & \max \left\{|2|^{n-1} \varphi\left(\frac{x}{2^{n}}, \frac{y}{2^{n}}\right),|2|^{n-1} \varphi\left(\frac{-x}{2^{n}}, \frac{-y}{2^{n}}\right)\right\}
\end{aligned}
$$

for all $x, y \in X \backslash\{0\}$. Taking the limit as $n \rightarrow \infty$ and using (2.8) and Lemma 2.1 we get $D T(x, y)=0$ for all $x, y \neq 0$ and so $T$ is a general quadratic mapping. Now we are going to prove the uniqueness of $T$. If $T^{\prime}$ is another general quadratic mapping satisfying (2.11) with $T^{\prime}(0)=f(0)$, then

$$
\begin{aligned}
T^{\prime}(x)-J_{k} T^{\prime}(x)= & \sum_{j=0}^{k-1}\left(\left(2^{2 j-1}+2^{j-1}\right) D T^{\prime}\left(\frac{x}{2^{j+1}}, \frac{-x}{2^{j+1}}\right)\right. \\
& \left.+\left(2^{2 j-1}-2^{j-1}\right) D T^{\prime}\left(\frac{-x}{2^{j+1}}, \frac{x}{2^{j+1}}\right)\right) \\
= & 0
\end{aligned}
$$

for any $k \in \mathbb{N}$ and so 


$$
\begin{aligned}
& \left\|T(x)-T^{\prime}(x)\right\| \\
& =\lim _{k \rightarrow \infty}\left\|J_{k} T(x)-J_{k} T^{\prime}(x)\right\| \\
& \leq \lim _{k \rightarrow \infty} \max \left\{\left\|J_{k} T(x)-J_{k} f(x)\right\|,\left\|J_{k} f(x)-J_{k} T^{\prime}(x)\right\|\right\} \\
& \leq \lim _{k \rightarrow \infty}|2|^{k-1} \max \left\{\left\|T\left(\frac{x}{2^{k}}\right)-f\left(\frac{x}{2^{k}}\right)\right\|,\left\|T\left(-\frac{x}{2^{k}}\right)-f\left(-\frac{x}{2^{k}}\right)\right\|,\right. \\
& \leq \lim _{k \rightarrow \infty}|2|^{k-1} \tilde{\varphi}\left(\frac{x}{2^{k}}\right) \\
& =0
\end{aligned}
$$

for all $x \in X \backslash\{0\}$. Since $T\left(0=f(0)=T^{\prime}(0)\right.$, we get $T(x)=T^{\prime}(x)$ for all $x \in X$. This completes the proof of the uniqueness of $T$.

Corollary 2.5. Let $r<1$ be a real number and $|2|<1$. If $f: X \rightarrow$ $Y$ satisfies the inequality

$$
\|D f(x, y)\| \leq \theta\left(\|x\|^{r}+\|y\|^{r}\right)
$$

for all $x, y \in X \backslash\{0\}$, then there exists a unique general quadratic mapping $T: X \rightarrow Y$ such that

$$
\|f(x)-T(x)\| \leq 2 \theta|2|^{-1-r}\|x\|^{r}
$$

for all $x \in X \backslash\{0\}$ with $T(0)=f(0)$.

Proof. Let $\varphi(x, y)=\theta\left(\|x\|^{r}+\|y\|^{r}\right)$. Since $|2|<1$ and $1-r>0$, we get

$$
\lim _{n \rightarrow \infty}|2|^{n} \varphi\left(2^{-n} x, 2^{-n} y\right)=\lim _{n \rightarrow \infty}|2|^{n(1-r)} \varphi(x, y)=0
$$

for all $x, y \in X \backslash\{0\}$. Therefore the conditions of Theorem 2.4 are fulfilled and it is easy to see that $\tilde{\varphi}(x)=2 \theta|2|^{-1-r}\|x\|^{r}$. By Theorem 2.4 there is a unique general quadratic mapping $T: X \rightarrow Y$ satisfying (2.14) with $T(0)=f(0)$.

Theorem 2.6. Let $\varphi:(X \backslash\{0\})^{2} \rightarrow[0, \infty)$ be a function such that

$$
\lim _{n \rightarrow \infty}|4|^{n} \varphi\left(2^{-n} x, 2^{-n} y\right)=0
$$

and

$$
\lim _{n \rightarrow \infty} \frac{\varphi\left(2^{n} x, 2^{n} y\right)}{|2|^{n}}=0
$$


for all $x, y \in X \backslash\{0\}$ and let for each $x \in X \backslash\{0\}$ the limit

$$
\begin{gathered}
\lim _{n \rightarrow \infty} \max _{0 \leq j<n}\left\{|2|^{2 j-1} \varphi\left(\frac{x}{2^{j+1}}, \frac{-x}{2^{j+1}}\right),|2|^{2 j-1} \varphi\left(\frac{-x}{2^{j+1}}, \frac{x}{2^{j+1}}\right),\right. \\
\left.\frac{\varphi\left(2^{j} x,-2^{j} x\right)}{|2|^{j+2}}, \frac{\varphi\left(-2^{j} x, 2^{j} x\right)}{|2|^{j+2}}\right\},
\end{gathered}
$$

denoted by $\tilde{\varphi}(x)$, exists. Suppose that $f: X \rightarrow Y$ is a mapping satisfying the inequality

$$
\|D f(x, y)\| \leq \varphi(x, y)
$$

for all $x, y \in X \backslash\{0\}$. Then there exists a unique general quadratic mapping $T: X \rightarrow Y$ such that

$$
\|f(x)-T(x)\| \leq \tilde{\varphi}(x)
$$

for all $x \in X \backslash\{0\}$ with $T(0)=f(0)$. In particular, $T$ is given by

$$
\begin{aligned}
T(x)= & \lim _{n \rightarrow \infty} \frac{4^{n}}{2}\left(f\left(\frac{x}{2^{n}}\right)+f\left(\frac{-x}{2^{n}}\right)-2 f(0)\right) \\
& +\lim _{n \rightarrow \infty} \frac{f\left(2^{n} x\right)-f\left(-2^{n} x\right)}{2^{n+1}}+f(0)
\end{aligned}
$$

for all $x \in X$.

Proof. Let $J_{n} f: X \rightarrow Y$ be a mapping defined by

$$
\begin{aligned}
J_{n} f(x)= & \lim _{n \rightarrow \infty} \frac{4^{n}}{2}\left(f\left(2^{-n} x\right)+f\left(-2^{-n} x\right)-2 f(0)\right) \\
& +\frac{f\left(2^{n} x\right)-f\left(-2^{n} x\right)}{2^{n+1}}+f(0)
\end{aligned}
$$

for all $x \in X$ and $n \in \mathbb{N}$. Notice that $J_{0} f(x)=f(x)$ and

$$
\begin{gathered}
\left\|J_{j} f(x)-J_{j+1} f(x)\right\| \\
=\| 2^{2 j-1} D f\left(\frac{x}{2^{j+1}}, \frac{-x}{2^{j+1}}\right)+2^{2 j-1} D f\left(\frac{-x}{2^{j+1}}, \frac{x}{2^{j+1}}\right) \\
-\frac{D f\left(2^{j} x,-2^{j} x\right)}{2^{j+2}}+\frac{D f\left(-2^{j} x, 2^{j} x\right)}{2^{j+2}} \| \\
\leq \max \left\{|2|^{2 j-1} \varphi\left(\frac{x}{2^{j+1}}, \frac{-x}{2^{j+1}}\right), \mid 2^{2 j-1} \varphi\left(\frac{-x}{2^{j+1}}, \frac{x}{2^{j+1}}\right),\right. \\
\left.\frac{\varphi\left(2^{j} x,-2^{j} x\right)}{|2|^{j+2}}, \frac{\varphi\left(-2^{j} x, 2^{j} x\right)}{\mid 2^{j+2}}\right\}
\end{gathered}
$$

for all $x \in X \backslash\{0\}$ and all $j \geq 0$. It follows from (2.15), (2.16) and (2.20) that the sequence $\left\{J_{n} f(x)\right\}$ is Cauchy for all $x \in X \backslash\{0\}$. Since $Y$ is 
complete and $J_{n} f(0)=f(0)$ for all $n \in \mathbb{N}$, we conclude that $\left\{J_{n} f(x)\right\}$ is convergent for all $x \in X$. Set

$$
T(x):=\lim _{n \rightarrow \infty} J_{n} f(x) .
$$

From (2.20) we have

$$
\begin{gathered}
\left\|J_{n} f(x)-f(x)\right\| \leq \max _{0 \leq j<n}\left\{|2|^{2 j-1} \varphi\left(\frac{x}{2^{j+1}}, \frac{-x}{2^{j+1}}\right),|2|^{2 j-1} \varphi\left(\frac{-x}{2^{j+1}}, \frac{x}{2^{j+1}}\right),\right. \\
\left.\frac{\varphi\left(2^{j} x,-2^{j} x\right)}{|2|^{\mid j+2}}, \frac{\varphi\left(-2^{j} x, 2^{j} x\right)}{|2|^{j+2}}\right\}
\end{gathered}
$$

for all $n \in N$ and all $x \in X \backslash\{0\}$. By taking $n$ to approach infinity in (2.21) and using (2.17) one obtains (2.19). By using (2.18) we get

$$
\begin{aligned}
&\left\|D J_{n} f(x, y)\right\|= \| \frac{D f\left(2^{n} x, 2^{n} y\right)-D f\left(-2^{n} x,-2^{n} y\right)}{2^{n+1}} \\
&+2^{2 n-1} D f\left(\frac{x}{2^{n}}, \frac{y}{2^{n}}\right)+2^{2 n-1} D f\left(\frac{-x}{2^{n}}, \frac{-y}{2^{n}}\right) \| \\
& \leq \max \left\{\frac{\varphi\left(2^{n} x, 2^{n} y\right)}{|2|^{n+1}}, \frac{\varphi\left(-2^{n} x,-2^{n} y\right)}{|2|^{n+1}},\right. \\
&\left.|2|^{2 n-1} \varphi\left(\frac{x}{2^{n}}, \frac{y}{2^{n}}\right),|2|^{2 n-1} \varphi\left(\frac{-x}{2^{n}}, \frac{-y}{2^{n}}\right)\right\}
\end{aligned}
$$

for all $x, y \in X \backslash\{0\}$ and all $n \in \mathbb{N}$. Taking the limit as $n \rightarrow \infty$ and using (2.15), (2.16) and Lemma 2.1 we get $D T(x, y)=0$ for all $x, y \neq 0$ and so $T$ is a general quadratic mapping. Now we are going to prove the uniqueness of $T$. Assume that $T^{\prime}$ is another general quadratic mapping satisfying (2.19) with $T^{\prime}(0)=f(0)$. Then

$$
\begin{aligned}
T^{\prime}(x)= & \sum_{j=0}^{k-1}\left(2^{2 j-1} D T^{\prime}\left(\frac{x}{2^{j+1}}, \frac{-x}{2^{j+1}}\right)+2^{2 j-1} D T^{\prime}\left(\frac{-x}{2^{j+1}}, \frac{x}{2^{j+1}}\right)\right. \\
& \left.-\frac{D T^{\prime}\left(2^{j} x,-2^{j} x\right)}{2^{j+2}}+\frac{D T^{\prime}\left(-2^{j} x, 2^{j} x\right)}{2^{j+2}}\right)+J_{k} T^{\prime}(x) \\
= & J_{k} T^{\prime}(x)
\end{aligned}
$$

for any $k \in \mathbb{N}$ and so 


$$
\begin{aligned}
& \left\|T(x)-T^{\prime}(x)\right\| \\
& =\lim _{k \rightarrow \infty}\left\|J_{2 k} T(x)-J_{2 k} T^{\prime}(x)\right\| \\
& \leq \lim _{k \rightarrow \infty} \max \left\{\left\|J_{2 k} T(x)-J_{2 k} f(x)\right\|,\left\|J_{2 k} f(x)-J_{2 k} T^{\prime}(x)\right\|\right\} \\
& \leq \lim _{k \rightarrow \infty} \max \left\{\frac{\left\|T\left(2^{2 k} x\right)-f\left(2^{2 k} x\right)\right\|}{|2|^{2 k+1}}, \frac{\left\|T\left(-2^{2 k} x\right)-f\left(-2^{2 k} x\right)\right\|}{|2|^{2 k+1}},\right. \\
& \frac{\left\|f\left(2^{2 k} x\right)-T^{\prime}\left(2^{2 k} x\right)\right\|}{|2|^{2 k+1}}, \frac{\left\|f\left(-2^{2 k} x\right)-T^{\prime}\left(-2^{2 k} x\right)\right\|}{|2|^{2 k+1}}, \\
& |2|^{4 k-1}\left\|T\left(\frac{x}{2^{2 k}}\right)-f\left(\frac{x}{2^{2 k}}\right)\right\|,|2|^{4 k-1}\left\|T\left(-\frac{x}{2^{2 k}}\right)-f\left(-\frac{x}{2^{2 k}}\right)\right\|, \\
& \left.|2|^{4 k-1}\left\|f\left(\frac{x}{2^{2 k}}\right)-T^{\prime}\left(\frac{x}{2^{2 k}}\right)\right\|,|2|^{4 k-1}\left\|f\left(-\frac{x}{2^{2 k}}\right)-T^{\prime}\left(-\frac{x}{2^{2 k}}\right)\right\|\right\} \\
& \leq \lim _{k \rightarrow \infty} \lim _{n \rightarrow \infty} \max _{0 \leq j<n}\left\{|2|^{2 j-2 k-2} \varphi\left(\frac{2^{2 k} x}{2^{j+1}}, \frac{-2^{2 k} x}{2^{j+1}}\right)\right. \text {, } \\
& |2|^{2 j-2 k-2} \varphi\left(\frac{-2^{2 k} x}{2^{j+1}}, \frac{2^{2 k} x}{2^{j+1}}\right) \frac{\varphi\left(2^{2 k+j} x,-2^{2 k+j} x\right)}{|2|^{2 k+j+3}}, \\
& \frac{\varphi\left(-2^{2 k+j} x, 2^{2 k+j} x\right)}{|2|^{2 k+j+3}},|2|^{4 k+2 j-2} \varphi\left(\frac{x}{2^{2 k+j+1}}, \frac{-x}{2^{2 k+j+1}}\right), \\
& |2|^{4 k+2 j-2} \varphi\left(\frac{-x}{2^{2 k+j+1}}, \frac{x}{2^{2 k+j+1}}\right) \text {, } \\
& \left.\frac{\varphi\left(2^{j-2 k} x,-2^{j-2 k} x\right)}{|2|^{j-4 k+3}}, \frac{\varphi\left(-2^{j-2 k} x, 2^{j-2 k} x\right)}{|2|^{j-4 k+3}}\right\}
\end{aligned}
$$

for all $x \in X \backslash\{0\}$ and all $k \in \mathbb{N}$. On the other hand, we have the inequalties

$$
\begin{gathered}
\lim _{k \rightarrow \infty} \lim _{n \rightarrow \infty} \max _{0 \leq j<n}\left\{|2|^{2 j-2 k-2} \varphi\left(\frac{2^{2 k} x}{2^{j+1}}, \frac{-2^{2 k} x}{2^{j+1}}\right)\right\} \\
\leq \lim _{k \rightarrow \infty} \lim _{n \rightarrow \infty} \max \left\{\max _{0 \leq j<k}\left\{|2|^{2 j-2 k-2} \varphi\left(\frac{2^{2 k} x}{2^{j+1}}, \frac{-2^{2 k} x}{2^{j+1}}\right)\right\},\right. \\
\max _{k \leq j<2 k}\left\{|2|^{2 j-2 k-2} \varphi\left(\frac{2^{2 k} x}{2^{j+1}}, \frac{-2^{2 k} x}{2^{j+1}}\right)\right\}, \\
\left.\max _{2 k \leq j<n}\left\{|2|^{2 j-2 k-2} \varphi\left(\frac{2^{2 k} x}{2^{j+1}}, \frac{-2^{2 k} x}{2^{j+1}}\right)\right\}\right\}
\end{gathered}
$$




$$
\begin{aligned}
& \leq \lim _{k \rightarrow \infty} \max \left\{|2|^{-4} \max _{k \leq j<2 k}\left\{\frac{\varphi\left(2^{j} x, 2^{j} x\right)}{|2|^{j}}\right\},|2|^{k-4} \max _{0 \leq j<k}\left\{\frac{\varphi\left(2^{j} x, 2^{j} x\right)}{|2|^{j}}\right\},\right. \\
& \left.\qquad|2|^{2 k-2} \lim _{n \rightarrow \infty} \max _{0 \leq j<n-2 k}\left\{|4|^{j} \varphi\left(\frac{x}{2^{j+1}}, \frac{-x}{2^{j+1}}\right)\right\}\right\} \\
& =0,
\end{aligned}
$$

$\lim _{k \rightarrow \infty} \lim _{n \rightarrow \infty} \max _{0 \leq j<n}\left\{\frac{\varphi\left(2^{2 k+j} x,-2^{2 k+j} x\right)}{|2|^{2 k+j+3}}\right\}$
$\leq \lim _{k \rightarrow \infty} \lim _{n \rightarrow \infty} \max _{2 k j<n-2 k}\left\{\frac{\varphi\left(2^{j} x,-2^{j} x\right)}{|2|^{j+3}}\right\}$

$=0$,

$$
\begin{aligned}
& \lim _{k \rightarrow \infty} \lim _{n \rightarrow \infty} \max _{0 \leq j<n}\left\{|2|^{4 k+2 j-2} \varphi\left(\frac{-x}{2^{2 k+j+1}}, \frac{x}{2^{2 k+j+1}}\right)\right\} \\
& \leq \lim _{k \rightarrow \infty} \lim _{n \rightarrow \infty} \max _{2 k \leq j<n-2 k}\left\{\left.|| 2\right|^{2 j-2} \varphi\left(\frac{-x}{2^{j+1}}, \frac{x}{2^{j+1}}\right)\right\} \\
& =0,
\end{aligned}
$$

$$
\begin{aligned}
& \lim _{k \rightarrow \infty} \lim _{n \rightarrow \infty} \max _{0 \leq j<n}\left\{\frac{\varphi\left(-2^{j-2 k} x, 2^{j-2 k} x\right)}{\left.|2|^{j-4 k+3}\right\}}\right. \\
& \leq \lim _{k \rightarrow \infty} \lim _{n \rightarrow \infty} \max \left\{\operatorname { m a x } _ { 0 \leq j < k } \left\{\frac{\varphi\left(-2^{j-2 k} x, 2^{j-2 k} x\right)}{\left.|2|^{j-4 k+3}\right\}},\right.\right. \\
& \max _{k \leq j<2 k}\left\{\frac{\varphi\left(-2^{j-2 k} x, 2^{j-2 k} x\right)}{\left.|2|^{j-4 k+3}\right\}},\right. \\
& \leq \max _{k \rightarrow \infty}\left\{\frac{\varphi\left(-2^{j-2 k} x, 2^{j-2 k} x\right)}{\left.|2|^{j-4 k+3}\right\}}\right\} \\
& |2|^{k-4} \max _{1 \leq j<k+1}\left\{|4|^{j} \varphi\left(\frac{x}{2^{j}}, \frac{-x}{2^{j}}\right)\right\}, \\
& |2|^{2 k-3} \max _{n \rightarrow 1 \leq j<2 k+1}\left\{\left.\right|^{j} \varphi\left(\frac{x}{2^{j}}, \frac{-x}{2^{j}}\right)\right\} \\
& \left.\max _{n \rightarrow j<n-2 k}\left\{\frac{\varphi\left(2^{j} x, 2^{j} x\right)}{|2|^{j}}\right\}\right\}
\end{aligned}
$$

$=0$ 
for all $x \in X \backslash\{0\}$ and all $k \in \mathbb{N}$. So the right hand side of (2.22) tends to 0 as $k \rightarrow \infty$. Since $T(0)=f(0)=T^{\prime}(0)$, we conclude that $T(x)=T^{\prime}(x)$ for all $x \in X$. This completes the proof of the uniqueness of $T$.

Corollary 2.7. Let $1<r<2$ be a real number and $|2|<1$. If $f: X \rightarrow Y$ satisfies the inequality

$$
\|D f(x, y)\| \leq \theta\left(\|x\|^{r}+\|y\|^{r}\right)
$$

for all $x, y \in X \backslash\{0\}$, then there exists a unique general quadratic mapping $T: X \rightarrow Y$ such that

$$
\|f(x)-T(x)\| \leq 2 \theta|2|^{-1-r}\|x\|^{r}
$$

for all $x \in X \backslash\{0\}$ with $T(0)=f(0)$. have

Proof. Let $\varphi(x, y)=\theta\left(\|x\|^{r}+\|y\|^{r}\right)$. Since $|2|<1$ and $1<r<2$, we and

$$
\lim _{n \rightarrow \infty}|4|^{n} \varphi\left(2^{-n} x, 2^{-n} y\right)=\lim _{n \rightarrow \infty}|2|^{n(2-r)} \varphi(x, y)=0
$$

$$
\lim _{n \rightarrow \infty} \frac{\varphi\left(2^{n} x, 2^{n} y\right)}{|2|^{n}}=\lim _{n \rightarrow \infty}|2|^{n(r-1)} \varphi(x, y)=0
$$

for all $x, y \in X$. Therefore the conditions of Theorem 2.6 are fulfilled and it is easy to see that $\tilde{\varphi}(x)=2 \theta|2|^{-1-r}\|x\|^{r}$. By Theorem 2.6 there is a unique general quadratic mapping $T: X \rightarrow Y$ satisfying (2.23) with $T(0)=f(0)$.

\section{References}

[1] T. Aoki, On the stability of the linear transformation in Banach spaces, J. Math. Soc. Japan 2 (1950), 64-66.

[2] I. S. Chang, E. H. Lee, and H. M. Kim, On Hyers-Ulam-Rassias stability of a quadratic functional equation, Math. Inequal. Appl. 6 (2003), 87-95.

[3] P. Găvruta, A generalization of the Hyers-Ulam-Rassias stability of approximately additive mappings, J. Math. Anal. Appl. 184 (1994), 431-436.

[4] D. H. Hyers, On the stability of the linear functional equation, Proc. Natl. Acad. Sci. U.S.A. 27 (1941), 222-224.

[5] S. S. Jin and Y. H. Lee, A fixed point approach to the stability of the generalized polynomial functional equation of degree 2, Commun. Korean Math. Soc. 28 (2013), 269-283.

[6] K. W. Jun and Y. H. Lee, A Generalization of the Hyers-Ulam-Rassias stability of the Pexiderized quadratic equations II, Kyungpook Math. J. 47 (2007), 91103.

[7] G. H. Kim, On the stability of functional equations with square-symmetric operation, Math. Inequal. Appl. 4 (2001), 257-266.

[8] H. M. Kim, On the stability problem for a mixed type of quartic and quadratic functional equation, J. Math. Anal. Appl. 324 (2006), 358-372. 
[9] Y. H. Lee, On the Hyers-Ulam-Rassias stability of the generalized polynomial function of degree 2, J. Chungcheong Math. Soc. 22 (2009), 201-209.

[10] Y. H. Lee, On the stability of the monomial functional equation, Bull. Korean Math. Soc. 45 (2008), 397-403.

[11] Y. H. Lee and K. W. Jun, A generalization of the Hyers-Ulam-Rassias stability of Jensen's equation, J. Math. Anal. Appl. 238 (1999), 305-315.

[12] Y. H. Lee and K. W. Jun, A generalization of the Hyers-Ulam-Rassias stability of Pexider equation, J. Math. Anal. Appl. 246 (2000), 627-638.

[13] Y. H. Lee and K. W. Jun, On the stability of approximately additive mappings, Proc. Amer. Math. Soc. 128 (2000), 1361-1369.

[14] M. S. Moslehian and Th. M. Rassias, Stability of functional equations in nonArchimedean spaces, Appl. Anal. Discrete Math. 1 (2007), 325-334.

[15] Th. M. Rassias, On the stability of the linear mapping in Banach spaces, Proc. Amer. Math. Soc. 72 (1978), 297-300.

[16] S. M. Ulam, A Collection of Mathematical Problems, Interscience, New York, 1960 .

$*$

Department of Mathematics Education

Gongju National University of Education

Gongju 314-060, Republic of Korea

E-mail: chjlee@gjue.ac.kr

$* *$

Department of Mathematics Education

Gongju National University of Education

Gongju 314-060, Republic of Korea

E-mail: lyhmzi@gjue.ac.kr 